\title{
THE COST OF INJURY IN NEW SOUTH WALES: A RISK MANAGEMENT FRAMEWORK
}

Mary Potter-Forbes

NSW Injury Risk Management Research Centre

University of New South Wales

The New South Wales Injury Risk Management Research Centre (IRMRC) at the University of New South Wales has been commissioned to estimate the NSW 'whole-oflife' cost of injury for use in future resource planning and investment decisions. This 'incidence based' approach estimates the expected real costs and economic losses for injuries sustained in a given year, calculated to the end point of recovery or death from the injury. The approach provides evidence of the long-term opportunity cost of 'doing nothing' to prevent the injury, and it may be distinguished from the 'prevalence based' approach that values the cost of new and old injuries in any year.

Problematically, the nature and severity of an injury, along with its treatment and sequelae, are usually complex and uncertain. Hence, the data requirements for a thorough 'bottom-up' approach to conducting a costing of injury are onerous and inevitably require the introduction of structured assumptions to produce a result in a costeffective fashion. This article describes the basic methodological issues associated with identifying and quantifying certain direct costs of the treatment of injury. A glossary of terms used in the article is provided in the box below.

\section{THETOTAL ECONOMIC COST OF INJURY AND THE AVOIDANCE OF COST SHIFTING}

Economic costing at the societal level is undertaken to demonstrate the wide-ranging cost effect of an injury on the victim, the family, and the broader society-not just on the health care system. If only the direct health and treatment expenditures are accounted for, then opaque cost shifting can occur. Therefore, in estimating the full economic cost of injury, one is valuing not only the opportunity costs associated with resources consumed in the treatment and maintenance of injury victims, but also the stream of societal and personal losses associated with reduced productivity, valued by estimating lost future earnings, and compromised quality of life - that is, the resultant pain and suffering and reduction in role performance due to disability and handicap. These losses jointly represent the value of a statistical life (VOSL), and in the literature it is these losses that represent by far the greatest proportion of societal costs. ${ }^{1,2}$

In the estimates of the cost of injury to Victoria for 199394, direct costs accounted for only 29.4 per cent of total cost while the indirect cost of mortality and morbidity on productivity alone, as calculated using the human capital approach, represented 71.6 per cent of the total societal cost. ${ }^{3}$ Near identical findings were reported by Rice et al., in a study published in the United States in 1989, who

\section{GLOSSARY OF TERMS \\ Bottom-up methodology}

Estimated actual expenditure from unit cost and volume data.

\section{Top-down methodology}

Known funding outlays allocated to the relevant cost units by some method other than an actual count.

\section{Sensitivity analysis}

Testing the parameters in a model with different values, for instance observing the effect of changes in the discount rate.

\section{Human capital approach}

Valuing economic losses in terms of lost productivity-average wage rates used as proxy for the lost productivity.

\section{Decision points}

The site of choice leading to different pathways that have different cost implications and potential outcomes.

\section{Attribution fraction}

Algorithm to allocate aggregate funds to cost centres, however defined.

\section{Diagnosis related groups (DRGs)}

A classification sytem for grouping acute inpatient epidodes of care into groups that are clinically coherent, relatively homogenous in respect of resource utilisation, and mutually exclusive. The 661 groups provide a measure of the product or output of the hospital and thus provide a basis for funding and budgeting.

\section{Opaque cost shifting}

The transfer of costs is not apparent if the focus is limited to that of a particular accounting entity. For instance, NSW Health's expenditures alone do not account for the costs, both direct and indirect, that are borne by the community when there are changes in policy-for example, the effect on society of de-institutionalisation and hospital-in-thehome are not necessarily cost beneficial in a societal sense.

estimated that the proportions for all injuries was 29 per cent in direct costs and 71 per cent in indirect mortality and morbidity cost (Watson and Ozanne-Smith, 1997). ${ }^{3}$ 


\section{FRAMEWORK FOR ANALYSIS-A RISK MANAGEMENT APPROACH}

The 'bottom-up' methodology can be employed to guide economic evaluations and can also provide a proxy for the severity of the consequences of categories of types of injury. When this proxy for injury severity is combined with the likelihood of the event occurring - the incidence rate-a more comprehensive assessment of risk is generated and the effective management of population health risks is made more certain. In the 'bottom-up' methodology, the 'decision points' that affect costs can be identified, and a 'sensitivity analysis' can be conducted in economic evaluation and services planning processes. However, the precision of the estimates so derived is problematic, given the limitations of existing data collection systems, the inconsistency in methods of categorisation, and the paucity of relevant clinical pathways and outcomes research.

\section{COST-OF-INJURY STUDIES IN AUSTRALIA}

Despite the theoretical argument for a full economic cost of injury, there are few published studies in this area in Australia. The most complete work has been done at the Australian Institute of Health and Welfare (AIHW) and Monash University Accident Research Centre (MUARC). In the 1999 AIHW study, only the direct costs of injury, and musculoskeletal disorders (including disorders that result from non-injury causes), were estimated using a prevalence-based 'top-down' methodology in which a series of 'attribution fractions' were developed to allocate national health expenditure to constituent costs. ${ }^{4}$ For the same period, MUARC used an essentially 'bottom-up' methodology to estimate the cost of injury to Victoria. ${ }^{3}$ In that study, productivity losses in terms of lost future work were also estimated. There was no attempt to value the lost quality of life component. In NSW, Moller applied the MUARC proportions to NSW data. ${ }^{5}$

\section{METHODOLOGICAL CONSTRAINTS IN DIRECT COSTING: EPIDEMIOLOGY AND DATA LINKAGE}

The most important source of data used in the IRMRC study was the Inpatient Statistical Collection (ISC) of the NSW Department of Health. This administrative data set provides the details for each episode of care and the initiating cause for each injury related separation-the E-code of Chapter XX of the ICD-10AM. In NSW, subsequent and related admissions are not linked to the original admission by a unique identifier, so they cannot be traced over time. The more detailed databases of insurers, such as those available through WorkCover NSW and the Motor Accident Authority, can be used to infer the scale of the experience of injury in New South Wales.
However, there are limitations to the use of these databases. First, the demographic profiles (as well as the nature and severity of injuries) recorded are not representative of the whole NSW population, and statistical estimation is required to derive reasonably valid epidemiological rates. Second, insurance organisations do not necessarily cost episodes of care using the real incidence rate or by the average costs for diagnosis related groups (DRG) but rather through an actuarially-derived estimate for bulk payment. Third, the systems used to categorise the nature and severity of injury in these databases are not necessarily based on the ICD-10-AM system of the ISC. Indeed, the Motor Accident Authority uses a modified Abbreviated Injury Score to determine the level of severity, which in turn determine average costs.

\section{WHAT THE INPATIENT STATISTICAL COLLECTION DOESTELL US}

Nevertheless, an estimate of the direct costs to NSW Health has been made from the data available in the NSW ISC. The number of E-coded hospital separations for NSW in 1999 , for the initial admission only, was 213,520 cases at a DRG average cost of $\$ 911$ million (Table 1). This estimate was derived by aggregating each E-coded admission and summing the DRG costs - the average cost for the associated medical diagnosis of each case in the sub-categories. In the absence of clinical costing this is as detailed a 'bottom-up' estimate as is possible.

There are many questions raised by the large sum of public monies spent in the treatment of injury. The 70,000 cases classified as complications of care must, however, be viewed with caution. In disaggregation, less than one per cent of the cost of hospitalisation is attributable to medical and surgical misadventure (ICD-10-AM categories Y60Y69). The bulk of the cases, as well as 85 per cent of the cost, is attributable to complications that did not arise at the time of the intervention. However, without content analysis of actual medical records, the nature of these complications is unknown. Further, the figure is likely to be inflated, as only 60 per cent of the injury coded (Chapter XX ICD-10-AM) admissions have an associated nature of injury code (Chapter XIX ICD-10-AM). This implies that the injury event did not have an adverse outcome-but it is uncertain whether there was no effect or there was an aggravation of an existing medical condition. What is almost certain, however, is that these complications occurred in hospital.

If these 70,000 cases are excluded then the direct hospital costs for the initiating admission are significantly reduced to $\$ 416$ million or approximately nine per cent of hospital expenditure. The NSW Health budget in 1997-98, the latest year for which published figures are available, was around $\$ 4.6$ billion dollars. ${ }^{6}$ By far the largest cost 


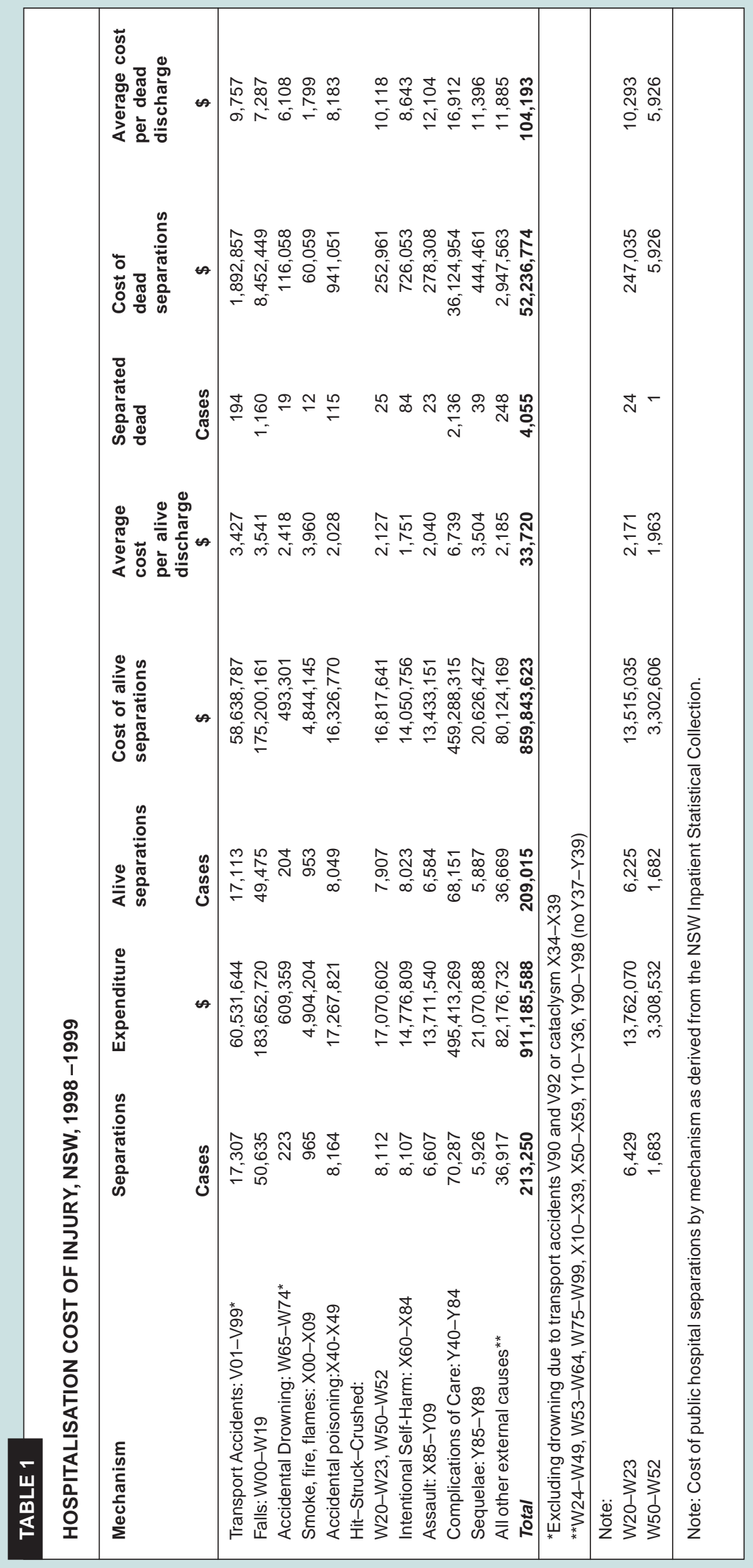


category is falls at around 51,000 separations and \$184 million in expenditure.

It is difficult to compare these figures with those from other studies. In the AIHW study, the direct costs of injury and musculoskeletal disorder (including disorders that result from non-injury causes) in Australia for the period 1993-94, using a 'top-down' methodology, were estimated at $\$ 5.603$ billion nationally. Although it is unclear as to the proportion of this sum attributable to NSW, as a third of the total Australian population is in NSW it is possible that as much as $\$ 1.68$ billion is expended here. In the Moller (2000) study, the total cost of direct morbidity in NSW in 1995-96 was estimated at $\$ 1.48$ billion. ${ }^{5}$ This evidence suggests that the cost estimates presented in Table 1 are plausible.

\section{CONCLUSION}

Due to limitations associated with data sets, we are only able to complete the epidemiological profiles to be used in assessments of cost from injuries by piecing together estimates from sources other than NSW population health data. This can be avoided through longitudinal studies of particular injury events for defined populations. The 'bottom-up' methodology developed for cost information will, therefore, be far more reliable than is currently the case; and, most importantly, from a risk management perspective it will be much more conducive to the implementation of risk management strategies.

\section{REFERENCES}

1. Miller T, Covington K, and Jensen A. Costs of injury by major cause, United States, 1995, cobbling together estimates. Measuring the Burden of Injuries. Mulder S and van Beeck EF (editors). Amsterdam: European Consumer Safety Association, Amsterdam, 1995; 23-40.

2. Elvik R. An analysis of official economic valuations of traffic accident fatalities in 20 motorized countries. Accid Annal Prev 1999; 27(2): 237-247.

3. Watson W and Ozanne-Smith J. The Cost of Injury to Victoria 1993-94. Melbourne: Monash University Accident Research Centre, Report no. 124, 1997.

4. Mathers C and Penm R. Health system costs of injury, poisoning and musculoskeletal disorders in Australia 199394. Canberra: Australian Institute of Health and Welfare, 1999. Catalogue no. 12.

5. Moller J. Patterns of Injury Costs NSW 1995-96. Adelaide: New Directions in Health and Safety, 2000.

6. Australian Institute of Health and Welfare. Australia's Health 2000, Canberra: Australian Institute of Health and Welfare, 2000. Catalogue no. 19.

\section{SAFE COMMUNITIES}

\section{Rebecca Mitchell \\ Injury Prevention and Policy Unit NSW Department of Health}

Safe Communities is a World Health Organization community-based model that offers communities a collaborative approach to managing injury prevention and safety promotion. Its key feature is the creation of a local infrastructure for addressing injury and safety priorities. This infrastructure is created through partnerships between stakeholders who share a vested interest in improving the standard of their community's safety. Local solutions are developed to address the local concerns about injuries, accidents, and safety. This approach to injury prevention encourages greater cooperation and collaboration between different levels of the business sector and government agencies and strives for a high level of community input.

The model has been successful because:

- a community defines its problems and identifies potential solutions to these problems;

- injury prevention and safety promotion efforts are coordinated at a regional level;
- it ensures that community interest groups are involved and support injury prevention or safety promotion projects;

- most importantly, it has been shown to lower the injury and accident rates in some communities.

\section{SAFE COMMUNITIES TRIALS IN NSW}

Trials of the Safe Communities model are being conducted in three locations in NSW. These pilot projects are a joint venture between the NSW Department of Health and the Roads and Traffic Authority (RTA). Pilot projects are being conducted in the following local government areas:

- Kempsey and Hastings (the Macleay-Hastings project);

- Gundagai;

- Kiama.

In Macleay-Hastings, the community is working on issues surrounding child injury, sporting injury, fall injury, alcohol consumption and injury, pedestrian safety, and car fleet safety. Injury prevention areas that are being considered in Gundagai are road safety, fall injury prevention, sports safety, and workplace safety. In Kiama, the community is working on issues surrounding home safety, fall injury prevention, alcohol consumption, 